\title{
Four-axis micro measuring systems performance verification
}

\author{
Giovanni Moroni (2), Stefano Petrò and Wahyudin P. Syam \\ Department of Mechanical Engineering, Politecnico di Milano, Via La Masa 1, 20156, Milan, Italy
}

Abstract:

This article describes the performance verification of 3D optical measuring instruments integrating a rotational axis using an artifact. The goal is the performance verification of four-axis measuring systems for the full 3D acquisition of micro-geometric parts. This type of measurement has many potential applications, such as in micro-tool, micro-mold, or micro-device manufacturing. The artifact is as simple as possible to reduce manufacturing costs, ensure easy calibration, comply with the ISO 10360 standard and consider all volumetric error contributions. The artifact may be useful to both measuring system manufacturers and users to provide a measurement traceability path.

Keywords: Performance, Measuring instrument, Micro metrology

\section{Introduction}

The current state of the art includes the ISO 10360 standard regarding procedures for coordinate measuring machine (CMM) performance verification. In particular, the ISO 10360-8 standard includes CMMs with optical distance sensors [1]. However, this part of the standard does not consider optical CMMs with a rotational axis, which are addressed in part 3 [2]. Optical microscopes, which can be considered optical CMMs for micro measurements, are gaining importance [3] as the application of micro-parts and micro-details become more widespread in everyday devices [4]. Non-contact measurements are preferable for micro-parts because physical contact has the potential to damage these delicate parts. However, to solve the accessibility issues that microscopes can present, a rotational axis is often needed. In spite of many emerging methods for non-contact micro measurements, specific performance verification methodologies are still lacking.

In this paper, reference artifacts that are used to evaluate the performance of 3D optical micro measuring instruments are presented. Additionally, the procedures for the performance verification by means of these artifacts are presented. The verification will consider the maximum error associated with the length measurement, as stated in ISO10360-8, and the maximum error associated with the rotational table (axis) of the instrument, as stated in IS010360-3. In this work, a 3D optical measurement instrument with a focal variation method and up to 4-axes measurement capability is used.

The contents of this paper are as follows: first, the description of the instrument used and the design of the proposed artifacts are presented in section 2 . The performance verification procedure and results are presented in section 3. Finally, conclusions are presented in section 4 .

\section{Innovative artifacts and calibration}

The instrument used for this verification program has the advantage of having an additional rotational axis (4-axis), allowing a full 3D measurement to be taken of any investigated parts. This functionality is very important, such as for the measurement of a micro-cutting tool. The instrument can be operated in two configurations: 3-axis (Cartesian, X-Y-Z) and 4axis ( 3 Cartesian +1 rotation). In the pure 3 -axis configuration, the total measuring volume is $100 \times 100 \times 100 \mathrm{~mm}$. A proposal for the artifact and the subsequent procedure for performance verification in this configuration have already been presented [5]. However, a reduction in measurement volume to $40 \times 40 \times 40 \mathrm{~mm}$ occurs if the additional rotational axis is used, as shown in Figure 1. This is a result of having to avoid collisions between the objective nosepiece and the rotational axis that may be caused if the measurement volume is too large.

In this paper, three types of artifacts are presented, denoted as artifacts 1, 2, and 3. The first two artifacts (artifacts 1 and 2) are used separately to evaluate the length measurement error and the rotation axis error. Artifact 3 combines the functionality of artifacts 1 and 2 and is used both for performance verification of the length measurement error and the rotation axis error in the reduced volume.

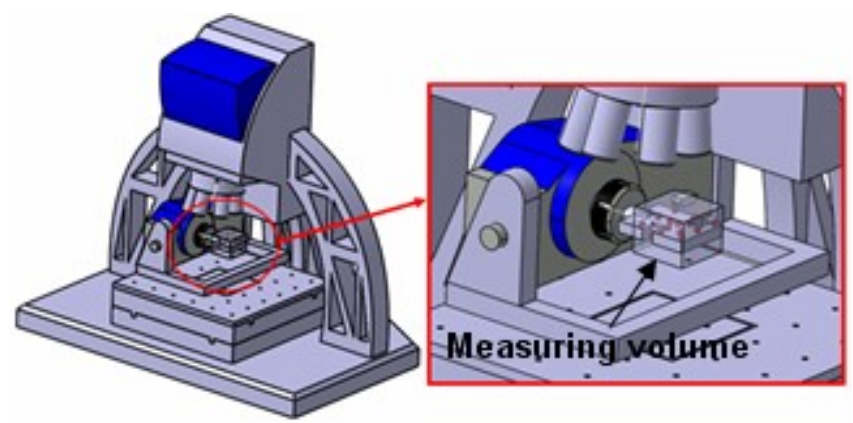

Figure 1. Focus variation instrument and its measuring volume in 4 -axis configuration.

\subsection{Artifact 1: Length measurement error}

Artifact 1 consists of an aluminum base plate, steel screws, and seven G5 grade steel ball bearings with a diameter of $5 \mathrm{~mm}$. The specifications for G5 grade ball bearings are set according to ISO $3290-1$ [6]. The size of the artifact is designed to be $40 \times 40 \times 40$ $\mathrm{mm}$, corresponding to the minimum measurement volume of the instrument. Bolts were used to increase the pre-load of the 
screws to augment their stiffness. Metal glue was used to fix the balls to the screw heads (see Figure 2). Balls with a $5 \mathrm{~mm}$ diameter were chosen because the image field of the lens used is smaller than the ball diameter. This is performed so that the image stitching process involved could be used to determine the center of the balls during the sphere feature association procedure. Adopting a $5 \mathrm{X}$ objective lens yields a field of view (FOV) equal to $2.8 \times 2.3 \mathrm{~mm}$ and a working distance of $23.5 \mathrm{~mm}$. This reduces the risk of collision between the artifact and the objective nosepiece.
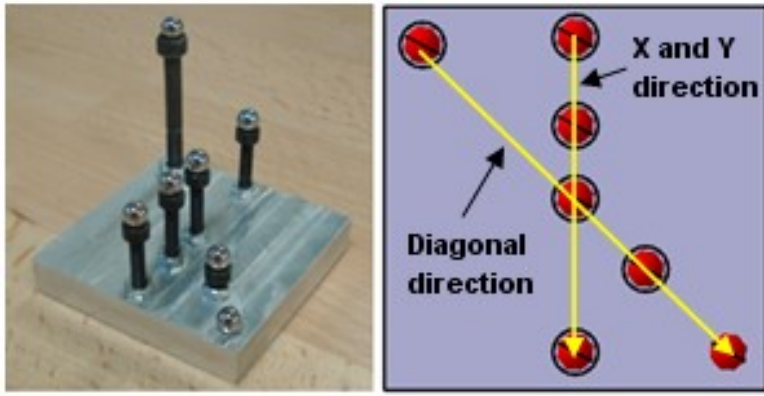

Figure 2. Artifact 1: length measurement error verification.

There are two ball position configurations: in-line and diagonal (see Figure 2, right). The in-line configuration allows the verification of performance along the $\mathrm{X}$ - or Y-axes, and the volumetric diagonal can be evaluated via the diagonal configuration. In each configuration, there are four balls with different reciprocal distances. Consequently, the minimum number of balls is used to provide five different length measurements for each configuration, as required in the ISO 10360-8 standard [1]. Each configuration has one ball in the center to use the minimum number of balls, reducing manufacturing cost and calibration time. Table 1 lists the reciprocal distances of the balls.

\subsection{Artifact 2: Rotation axis error}

Artifact 2 requires a simpler design. Only two $5 \mathrm{~mm}$ diameter G5 grade steel ball bearings are needed to fulfill the requirements of the ISO 10360-3 standard [2]. Spheres A and B are approximately located at an identical radius of $20 \mathrm{~mm}$, are diametrically opposite of each other and are positioned at different heights with respect to the artifact base. The artifact is made of aluminum to reduce the weight $(95 \mathrm{~g})$ due to the $2 \mathrm{~kg}$ maximum payload limit of the spindle. Artifact 2 is shown in Figure 3.
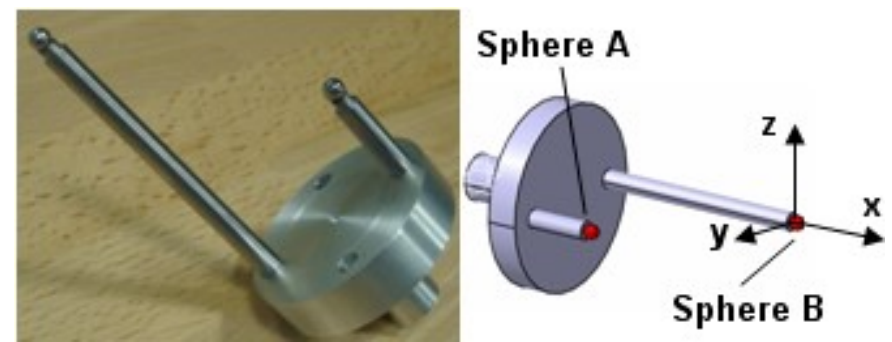

Figure 3. Artifact 2: rotation table (axis) error verification.

2.3. Artifact 3: Combination of length measurement error and rotation axis error evaluation

Artifact 3 combines the functionality of artifacts 1 and 2 so that the verification of the length measurement and rotation axis errors can be performed with a single artifact. The artifact has a size of $40 \times 40 \mathrm{~mm}$ with weight of $118 \mathrm{~g}$. It is composed of a rectangular aluminum plate mounted on a cylindrical gripper that is inserted into the spindle chuck. Spindle load limitations need to be considered. Fifteen G5 grade steel ball bearings with a diameter of $5 \mathrm{~mm}$ are used. Thirteen balls are glued to a prepared "seat" on the aluminum base and used for the length measurement error evaluation. The remaining two are glued onto screws and used for the rotation axis error evaluation. Artifact 2 is shown in Figure 4.

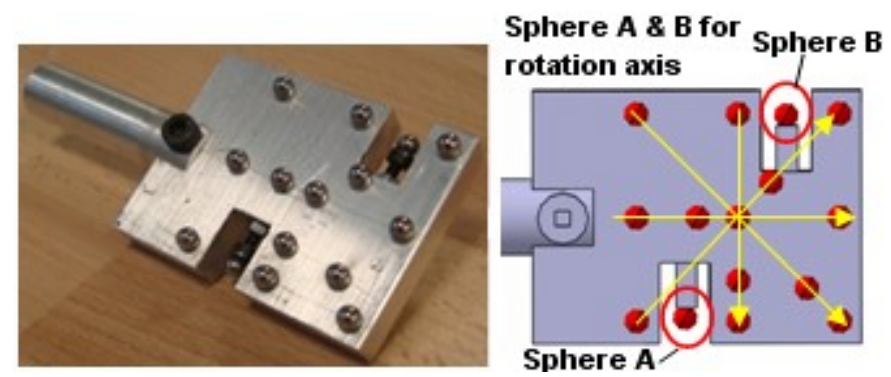

Figure 4. Artifact 3: innovative artifact for both performance verification of length measurement error and rotation axis error.

The thirteen balls shown in Figure 4 have four configurations: horizontal, vertical, diagonal 1 (from top-left to bottom-right), and diagonal 2 (from bottom-left to top-right). The horizontal and vertical configurations are used to evaluate the X- and Y-axes, respectively, whereas the other two diagonal configurations are used to evaluate the volumetric diagonal of the measurement volume by rotating the artifact $+45^{0}$ and $-45^{0}$. In each configuration, four balls are separated at different distances, similarly to artifact 1 . Table 1 lists the five different lengths used in each configuration. The two balls on the sides are used for the rotation axis error evaluation. As required by the ISO 10360-3 standard [2], these balls are approximately located at a radius of $20 \mathrm{~mm}$, are diametrically opposite and are at different distances from the chuck.

Notably, a reference flat plane could be easily manufactured on this artifact to fulfill the requirements of the flat form measurement test in ISO 10360-8.

Table 1. List of length distances for artifacts 1 and 3 with their calibration uncertainty expressed according to the GUM.

\begin{tabular}{|c|l|l|}
\hline Artifact & \multicolumn{1}{|c|}{ Configuration } & \multicolumn{1}{|c|}{ Length [mm } \\
\hline Artifact 1 & In-line & $9.3523(2) ; 11.7205(2) ;$ \\
& & $20.0393(3) ; 29.3908(1) ;$ \\
& & $41.1065(1)$ \\
\hline Artifact 1 & Diagonal & $12.3996(1) ; 20.8125(1) ;$ \\
& & $30.9691(2) ; 51.7087(1) ;$ \\
& & $64.10263(1)$ \\
\hline Artifact 3 & Horizontal & $8.5638(2) ; 11.9611(2) ;$ \\
& & $19.9900(3) ; 20.5243(1) ;$ \\
& & $40.5142(1)$ \\
\hline Artifact 3 & Vertical & $8.0663(1) ; 12.5476(1) ;$ \\
& & $19.8484(3) ; 32.3944(1) ;$ \\
& & $9.4602(1)$ \\
\hline Artifact 3 & Diagonal 1 & $28.6665(1) ; 48.0651(1) ;$ \\
& & $57.3362(1)$ \\
\hline Artifact 3 & Diagonal 2 & $9.5514(1) ; 18.7434(3) ;$ \\
& & $28.9887(1) ; 38.5335(1) ;$ \\
& & $57.2725(1)$ \\
\hline
\end{tabular}

\subsection{Artifact calibration}

The distance between the two spheres centers (measurand) in artifacts 1 and 3 are calibrated for the length measurement error evaluation (table 1). For this calibration, a tactile CMM with $\mathrm{E}_{0, \mathrm{MPE}}$ $=2+\mathrm{L} / 300 \mu \mathrm{m}$ [1] was used. The coordinates of the centers of the balls, obtained by fitting a Gaussian sphere to the points probed by the CMM, allow the calculation of reciprocal distances between the balls. A multiple measurement strategy was used and the uncertainties in the calibration are determined by varying the orientation of the artifact. The key principle of this procedure is that the volumetric error of the CMM is taken into account when the measurements are conducted in different orientations.

Four different orientations were selected. In each of these four orientations, five measurements were conducted such that the repeatability contribution of the calibration can be estimated and included in the total calibration uncertainty. One point that should be carefully addressed is the selection of the four 
orientations. The selection of the orientation is chosen such that all the balls have a different position in the different orientations. Because of this reason, each orientation is skewed to follow this requirement (Figure 5). The compensation to the scale error is achieved by considering the measurement of an additional reference calibrated artifact (in this case, a $150 \mathrm{~mm}$ gauge block). The calibration expanded uncertainty obtained for the distance between two sphere centers in artifact 1 is between $300 \mathrm{~nm}$ and $800 \mathrm{~nm}$ and is between $200 \mathrm{~nm}$ and $700 \mathrm{~nm}$ for artifact 3, depending on the length considered. From Table 1, uncertainties smaller than the instrument E0,MPE can be obtained. This is due to three characteristics of the procedure. First, by fitting the center of the spheres on several hundreds of points, the random error in the identification of the centers is reduced. Second, the scale error is reduced by the use of a reference artifact. Finally, 20 measurements were taken, and averaged.
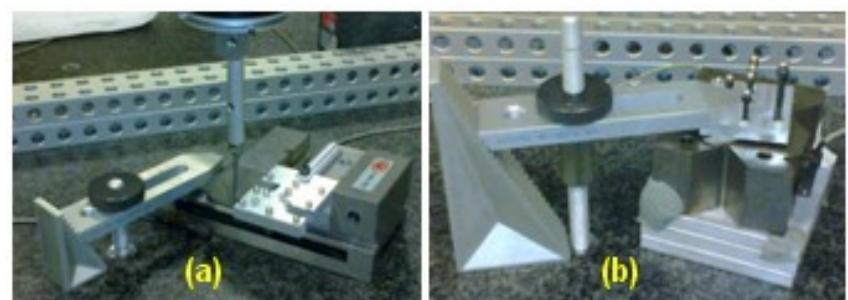

Figure 5.Skewed orientation in calibration (a) for artifact 3, and (b) for artifact 1.

\section{Performance verification}

\subsection{Length measurement error (ISO 10360-8)}

Artifacts 1 and 3 were used for the length measurement performance verification. In the verification procedure, the error is defined as a deviation in the distance between the two sphere centers obtained by the optical instrument from the calibrated distance values (as the conventional true values). The procedures are different for each artifact. Figure 10b shows the placement of artifact 1 . The procedure for artifact 1 is described as follows. First, position 1 is selected such that the in-line configuration of the balls is in the horizontal position, with respect to the operator's view. In this position, the length measurement evaluations are carried out for the $\mathrm{X}$-axis and for volumetric diagonal 1 (Top-Left to Bottom-Right). After that, the artifact is manually rotated $90^{\circ}$ (clock-wise) such that the in-line configuration of the balls is in the vertical position with respect to the operator's view. Length measurement evaluations are performed for the Y-axis and for diagonal 2 (Top-Right to BottomLeft). Subsequently, diagonal 3 (Bottom-Right to Top-Left) and diagonal 4 (Bottom-Left to Top-Right) can be evaluated by rotating the artifact $90^{\circ}$ (clock-wise).

The procedure for measuring artifact 3 is much simpler. With this artifact, operator involvement is only necessary to mount the artifact holder to the spindle chuck and to check the inclination angle (Figure 10c). For the X- and Y-axis length measurement evaluation, the artifact is set to an approximately flat position so that the verification can be performed. Volumetric errors for diagonals 1 and 2 are evaluated after rotating the spindle $-45^{0}$ (Figure 10a) before the verification of these diagonals is carried out. Finally, for diagonals 3 and 4, the verification is performed after rotating the spindle $+45^{\circ}$.

The measurement of the center of each ball required the stitching of four image fields, such that the stitching error is taken into account. After obtaining the ball's center location, the relative distance can be calculated. In every direction, the measurement is carried out reciprocally (left-to-right-to-left), such that the hysteresis of the stage is taken into account. From this, three measurements for each length type are obtained. The results from the performance verification in the X-direction are presented in Figure 6. In this direction, the maximum error obtained from artifacts 1 and 3 is approximately $4.25 \mu \mathrm{m}$ and
$3.18 \mu \mathrm{m}$, respectively. Figure 7 shows the verification results in the Y-direction. Considering these measurement results, artifact 3 gives maximum error of $4.8 \mu \mathrm{m}$, which is approximately $1.5 \mu \mathrm{m}$ higher than artifact 1.

Figure 8 presents error results for the diagonals. It is noted that artifact 3 contains two different diagonal configurations (see Table 1): two sets of errors are plotted. It is possible to note that the maximum error observed from the diagonal measurements is equal to $11.3 \mu \mathrm{m}$ and $11.8 \mu \mathrm{m}$ for artifacts 1 and 3, respectively. Finally, from Figure 9, which reports all measurements (along the $\mathrm{x}, \mathrm{y}$ and four diagonals) for both artifacts, it is worth noting that the dispersion of the errors seems to increase and that the error

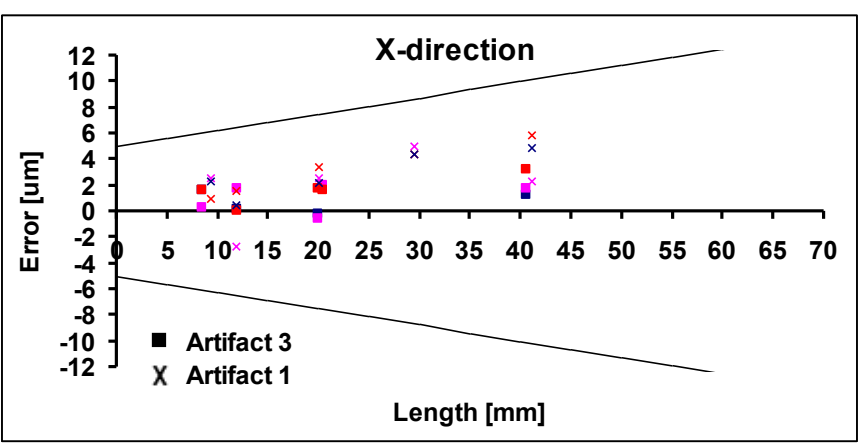

Figure 6.Length measurement errors in the X-direction (artifact 1 and 3).

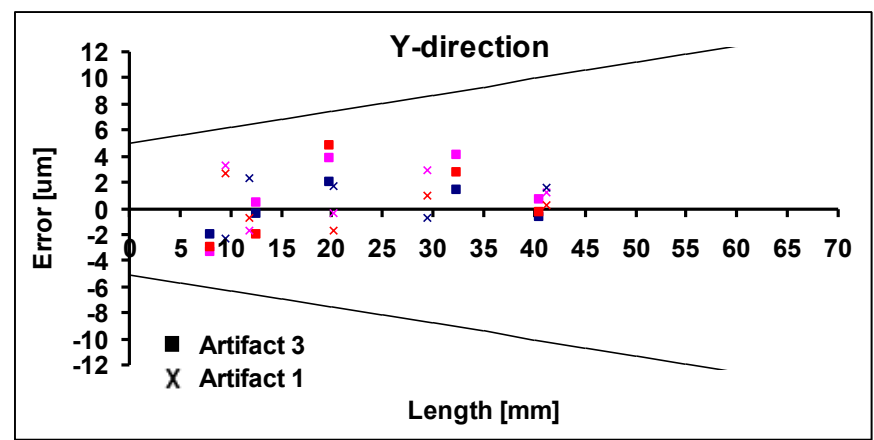

Figure 7.Length measurement errors in the Y-direction (artifact 1 and 3).

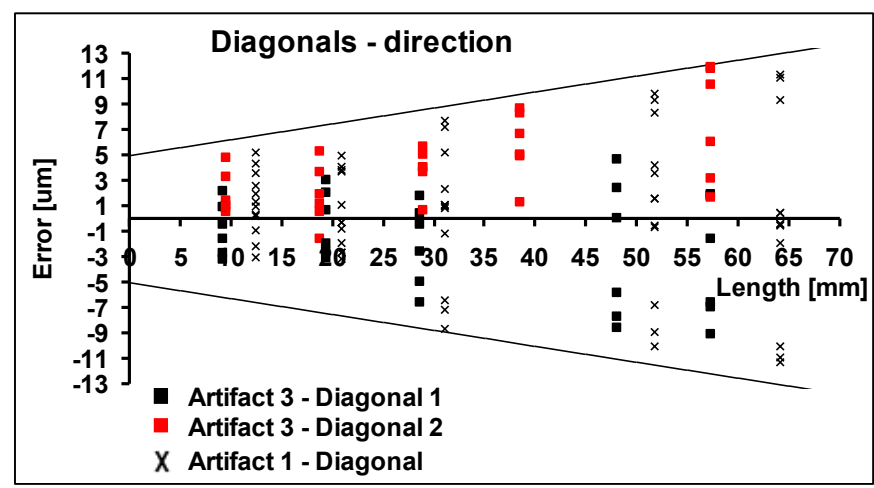

Figure 8.Length measurement errors in All 4 diagonals (artifact 1 and 3).

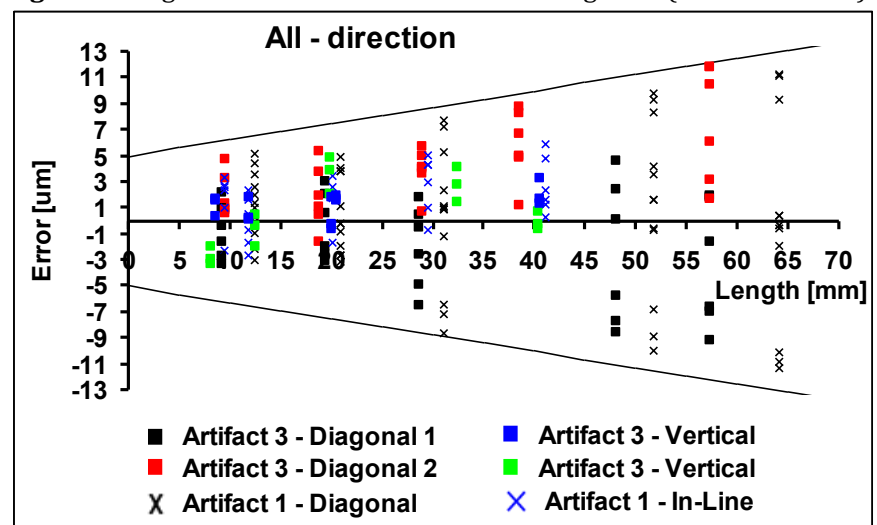

Figure 9.Length measurement errors for the $\mathrm{X}$-axis, Y-axis, and 4 diagonals (artifact 1 and 3 ).

always seems to be less than $\pm(5+L / 8) \mu \mathrm{m}$. 
In general, the results show good agreement between the two artifacts. The difference in the errors evaluated using artifacts 1 and 3 are caused by the different types of lengths among the balls that are measured. To confirm this conclusion, a KolmogorovSmirnov test was conducted to compare the statistical distribution of the length measurement errors carried out on artifacts 1 and 3 . The resulting p-value of 0.78 suggests there is no evidence to refuse the null hypothesis that the distributions are identical.

From these results, it is possible to evaluate the performance of the system as well. By considering a probing size error of $P_{\text {Size.Sph } 1 \times 25 ; \mathrm{Tr} ; \mathrm{ODS}}=2 \mu \mathrm{m}$ and a probing form error of $P_{\text {Form.Sph } 1 \times 25 ; \mathrm{T} ; \mathrm{ODS}}=8 \mu \mathrm{m}$, we can state that the length measurement error is $E_{\mathrm{Bi} . \mathrm{Sph} 1 \times 25 ; \mathrm{Tr} ; \mathrm{ODS}, \mathrm{MPE}}= \pm(15+L / 8) \mu \mathrm{m}[1]$.

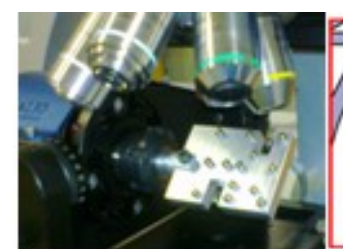

(a)

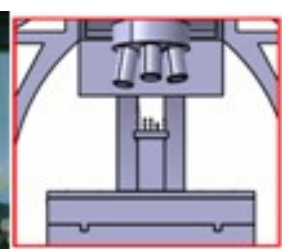

(b)

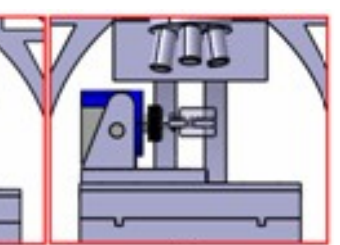

(c)
Figure 10.(a) Performance verification using artifact 3 rotated by $-45^{0}$, (b) placement of artifact 1 , and (c) placement of artifact 3.

\subsection{Rotation axis error (ISO 10360-3)}

The procedure used for the rotation axis performance verification conforms to ISO 10360-3 [2]. A slight difference between the procedures stated in [2] regards the determination of the reference. Due to the instrument's inherent characteristics, the reference is determined as follows (see Figure 3 right). The zero point is set at the center of sphere $\mathrm{B}$. The Z-axis is parallel to the lens axis. The $\mathrm{X}$-axis is along the spindle axis and the $\mathrm{Y}$-axis is set perpendicular to the $\mathrm{X}-\mathrm{Z}$ plane. A full $360^{\circ}$ rotation was evaluated for both artifacts. An unreachable position at a rotation position between $+45^{0}$ to $+90^{0}$ from the initial flat position $\left(0^{0}\right)$ was observed due to a collision between the objective nosepiece and the artifact. For each predetermined rotation angle, the center of each sphere is calculated by acquiring four images of the sphere and stitching them together.

A comparison of the results obtained from artifacts 2 and 3 in Figures 11, 12, and 13 shows the axis rotation error in the X-, Y-, and Z-direction, respectively. The maximum errors of each rotation angle are plotted. Errors at positive rotation angles were obtained from sphere A and errors at negative rotation angles were obtained from sphere B. From the performance verification of artifact 2, the maximum rotation axis errors for the $\mathrm{X}-, \mathrm{Y}-$, and Z-directions are approximately $5 \mu \mathrm{m}, 7.5 \mu \mathrm{m}$, and $14.7 \mu \mathrm{m}$, respectively. Additionally, for artifact 3 , the maximum errors obtained are approximately $7.6 \mu \mathrm{m}, 9.3 \mu \mathrm{m}$, and $17.3 \mu \mathrm{m}$ for the $\mathrm{X}-, \mathrm{Y}-$, and Z-directions, respectively. In both artifacts, the maximum errors are observed along the Z-direction. A slightly larger maximum error was observed in all directions for artifact 3 ranging from $1.8 \mu \mathrm{m}$ to $2.6 \mu \mathrm{m}$.

In general, the obtained errors from both artifacts show good agreement. To strengthen this conclusion, a Kolmogorov-Smirnov test was again conducted to compare the statistical distribution of the length measurement errors for artifacts 2 and 3 . The resulting p-value of 0.30 suggests that there is no evidence to refuse the null hypothesis that the distributions are identical.

Finally, the performance with a rotary axis is evaluated as follows: $\mathrm{MPE}_{\mathrm{FR}}=18 \mu \mathrm{m}, \mathrm{MPE}_{\mathrm{FT}}=10 \mu \mathrm{m}$, and $\mathrm{MPE}_{\mathrm{FA}}=8 \mu \mathrm{m}$.

\section{Conclusions}

In this work, reference artifacts along with procedures for the performance verification of a 3D optical micro measuring instrument using the focus variation method have been presented. The verification is conducted using two approaches. First, the performance verifications were conducted separately using two artifacts for each length measurement error (ISO10360-8) and rotation axis error (ISO10360-3). Secondly, a single hybrid artifact is used for both types of performance verification. The results show good agreement between the two approaches. The use of a single artifact is an improvement (allowing the possibility of checking the rotational and volumetric performance without manually changing the system), and a step forward in the evolution of verification procedures. The final goal is the development of an artifact and a procedure allowing the simultaneous verification of the volumetric and rotation accuracy.

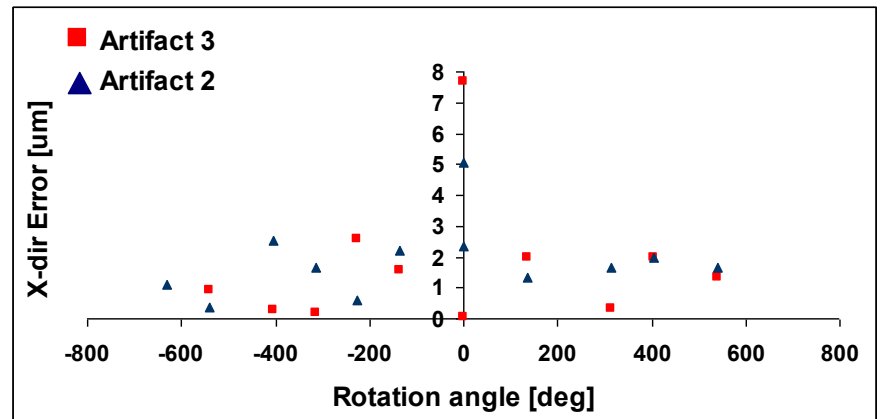

Figure 11.Rotation axis error in X-direction (artifact 2 and artifact 3 ).

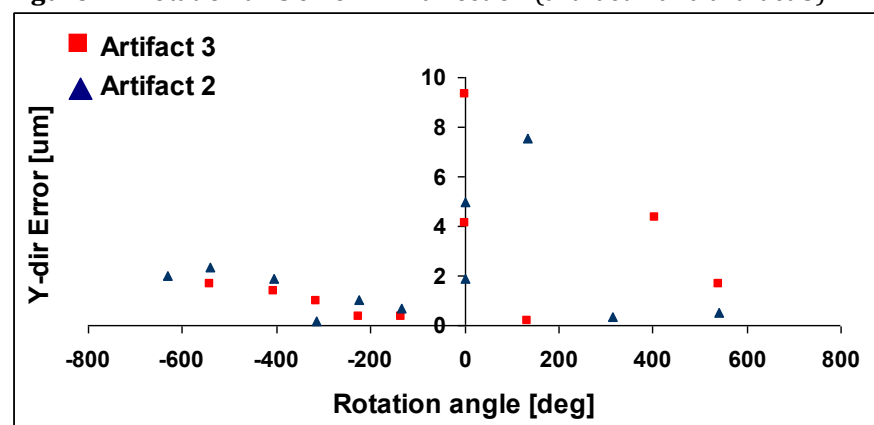

Figure 12.Rotation axis error in Y-direction (artifact 2 and artifact 3).

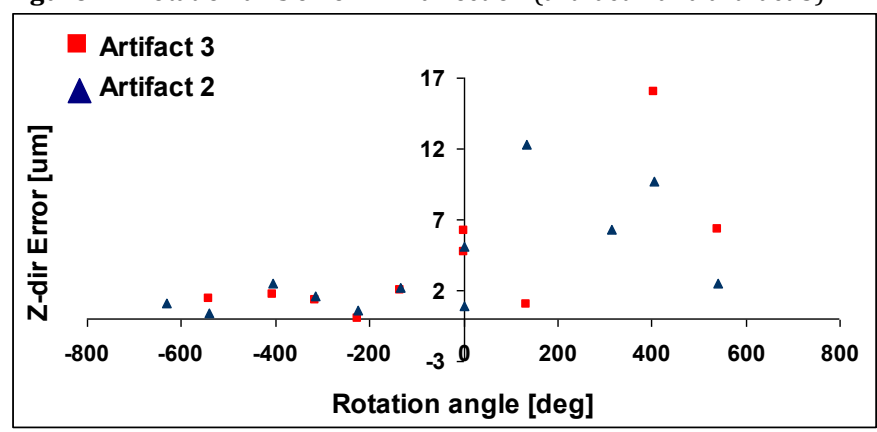

Figure 13.Rotation axis error in the Z-direction (artifacts 2 and 3).

\section{Acknowledgements}

Financial support for this work has been provided as part of project REMS "Rete Lombarda di Eccellenza per la Meccanica Strumentale e Laboratorio Esteso", funded by Lombardy Region (Italy), CUP: D81J10000220005.

\section{References}

[1] ISO 10360-8. (2013). GPS-Acceptance and re-verification tests for coordinate measuring systems (CMS) -Part 8: CMMs with optical distance sensors. Geneva, Switzerland.

[2] ISO 10360-3. (2007) GPS-Acceptance and re-verification tests for coordinate measuring systems (CMS) -Part 3: CMMs with rotation table. Geneva, Switzerland.

[3] Hansen, H. N., Carneiro, K., Haitjema, H., De Chiffre, L. (2006). "Dimensional Micro and Nano Metrology", CIRP Annals Manufacturing Technology, Vol. 55, No. 2, pp. 721-743.

[4] Alting, L., Kimura, F., Hansen, H. N., Bissacco, G. (2003). “Micro Engineering”, CIRP Annals: Manufacturing Technology, Vol. 52, No. 2, pp. 635-657.

[5]Moroni, G., Petrò, S., Syam, W. P. (2013). "On performance verification of 3D micro measuring instrument", 28 thASPE Annual Meeting, October 20-25, 2013, Saint Paul, Minnesota, USA.

[6] ISO 3290-1. (2008). Rolling bearings-Balls. Part 1: Steel balls. Geneva, Switzerland. 